OPEN ACCESS

Edited by:

Himadri Chattopadhyay

Jadavpur University, India

Reviewed by:

Arindam Bit,

National Institute of Technology

Raipur, India

Filippo Cademartiri,

Gabriele Monasterio Tuscany

Foundation (CNR), Italy

${ }^{*}$ Correspondence:

Chaoyang Wen

wenchaoyang@pkuih.edu.cn

Yisha Tong

yisha.tong@austin.org.au

tThese authors have contributed equally to this work

Specialty section:

This article was submitted to

Cardiovascular Imaging,

a section of the journal

Frontiers in Cardiovascular Medicine

Received: 06 December 2021 Accepted: 02 February 2022 Published: 24 February 2022

Citation:

Zhao R, Zheng $H$, Wang W, Du Y, Tong $Y$ and Wen $C$ (2022) Quantitative Evaluation of Post-stenotic Blood Flow Disturbance in Canine Femoral Artery Stenosis Model: An Early Experience With Vector Flow Imaging. Front. Cardiovasc. Med. 9:829825. doi: 10.3389/fcvm.2022.829825

\section{Quantitative Evaluation of Post-stenotic Blood Flow Disturbance in Canine Femoral Artery Stenosis Model: An Early Experience With Vector Flow Imaging}

\author{
Rui Zhao ${ }^{1 \dagger}$, Haining Zheng ${ }^{1 t}$, Wei Wang ${ }^{2 t}$, Yigang $D u^{3}$, Yisha Tong ${ }^{4 *}$ and Chaoyang Wen ${ }^{1 *}$ \\ ${ }^{1}$ Department of Ultrasound, Peking University International Hospital, Beijing, China, ${ }^{2}$ Department of Ultrasound, The Fourth \\ Medical Center of PLA General Hospital, Beijing, China, ${ }^{3}$ Shenzhen Mindray Bio-Medical Electronics Co., Ltd., Shenzhen, \\ China, ${ }^{4}$ Department of Vascular Surgery, Austin Hospital, University of Melbourne, Melbourne, VIC, Australia
}

Objective: To investigate the value of Vector Flow Imaging (V Flow) in the assessment of post-stenotic turbulence in the canine arterial stenosis model.

Materials and Methods: Canine femoral artery stenosis models were established using ameroid constrictors in 12 beagle dogs. 50\% and then 70\% femoral artery stenoses were confirmed by selective femoral artery angiography. $\vee$ Flow was used to measure femoral artery flow turbulence index (Tur) preoperatively as a baseline. After establishing of a $50 \%$ and then $70 \%$ stenoses, the Tur indices were recorded in the femoral artery at 1, 3, 5, 7, $9,11,13,15,17$, and $19 \mathrm{~mm}$ distal to the stenosis.

Results: Baseline Tur indices of normal canine femoral arteries were $<1 \%$ in 11 of 12 cases (91.7\%). Distal to a 50\% stenosis, the Tur index (>1\%) was recorded in 83.3-100\% cases between 1 and $9 \mathrm{~mm}, 41.7-58.3 \%$ between 11 and $17 \mathrm{~mm}$, and $16.7 \%$ at $19 \mathrm{~mm}$. For a $70 \%$ stenosis, the Tur index (>1\%) occurred in $81.8-100 \%$ cases between 1 and $17 \mathrm{~mm}$ distal to the stenosis, and $63.6 \%$ at $19 \mathrm{~mm}$. The Tur index peaked around $7 \mathrm{~mm}$ or 2.3 times of the initial vessel diameter $(3 \mathrm{~mm})$ downstream for a $50 \%$ stenosis and $11 \mathrm{~mm}$ or 3.7 times of vessel diameter downstream for a $70 \%$ stenosis.

Conclusion: V Flow with Tur index measurement adds quantitative information of post-stenotic turbulence when assessing an arterial stenosis with ultrasound. Tur index of $1 \%$ seems a useful threshold for assessment of flow turbulence in this small sample study. Further studies with larger sample size are needed to evaluate the value of $\vee$ Flow in clinical applications.

Keywords: arterial stenosis, Doppler, duplex ultrasound, femoral artery, post-stenotic turbulence, vector flow imaging 


\section{INTRODUCTION}

Arterial stenosis/occlusion is a common vascular pathology and most commonly caused by atherosclerosis $(1,2)$. Other causes of this condition include Takayasu's disease, Burger's disease (thromboangiitis obliterans), fibromuscular dysplasia, adventitial cystic disease of the popliteal artery, popliteal artery entrapment, endofibrosis of the external iliac artery (iliac artery syndrome in cyclists), thoracic outlet syndrome, etc. (2,3). Depending on the artery or arteries involved, arterial stenosis/occlusion may result in ischaemia of the brain, viscera, upper or lower limbs and cause relevant ischaemic symptoms.

Duplex ultrasound scanning has been widely used to evaluate arterial stenosis in various parts of the human body, including carotid, upper limb, visceral/renal, aortoiliac and lower limb arteries (4-6). Duplex ultrasound assessment of arterial stenosis includes observation of intraluminal plaque, measurement of velocities and evaluation of post-stenotic turbulence, as well as the profile of the waveform (7). Traditionally, flow turbulence is qualitatively assessed by the appearance of multiple colors on the color Doppler image and spectral broadening on the spectral Doppler waveform. On the spectral Doppler waveform, the spectral window is the black zone between the spectral line and the baseline. Spectral broadening is widening of the spectral line and filling of the spectral window (8). However, neither the color Doppler image nor the spectral Doppler waveform assessment is quantitative. Recently, high frame rate vector flow imaging (V Flow) has become available (9-11) and it is capable to quantitatively evaluate flow turbulence $(11,12)$. Most published studies using $\mathrm{V}$ Flow have been investigations on carotid arteries for blood flow visualization, wall shear stress measurement and volume flow estimation, as well as in the assessment of arterial stenosis before and after carotid artery stenting (13-21).

\section{MATERIALS AND METHODS}

The experiment study was performed between April 2020 and July 2020. The study was approved by the PLA General Hospital Medical Ethics Committee.

\section{Establishment of Canine Femoral Artery Stenosis Model}

Twelve beagle dogs aged 12-24 months were purchased from the Animal Experiment Centre of Nongnong (Beijing) Biotechnology Co. Ltd. (Beijing, China). Preoperative duplex ultrasound scanning was performed to confirm that internal diameters of the canine femoral arteries were $3.0 \pm 0.1 \mathrm{~mm}$.

The aim of this study is to explore the value of V Flow in the evaluation of post-stenotic turbulence in the canine arterial stenosis model.

Ameroid constrictors with inner diameters of $2.5 \mathrm{~mm}$ (Research Instruments SW, USA) were used to create canine femoral artery stenosis. An ameroid constrictor has an inner ring of casein that is surrounded by a stainless-steel sheath. Casein is a hygroscopic material that swells as it slowly absorbs body fluid and the stainless-steel sheath forces the casein to swell inwardly. This results in the internal diameter of the constrictor decreasing gradually over a few weeks.
Under general anesthesia, the right canine femoral artery in the groin was dissected and applied with $3 \mathrm{ml}$ lidocainepapaverine solution $(50 \mathrm{mg} / 2.5 \mathrm{ml}$ lidocaine and $15 \mathrm{mg} / 0.5 \mathrm{ml}$ papaverine) to prevent vasospasm. An ameroid constrictor ring was then placed over the artery.

An arterial duplex ultrasound scan was performed $4-5 \mathrm{~h}$ after the surgery to exclude femoral artery spasm. A selective right femoral artery angiogram with was then performed with 2 perpendicular projections via left femoral artery catherization (OEC 9900 Elite mobile C-arm imaging system, GE Healthcare, USA). The establishment of the $50 \%$ canine femoral artery stenosis using the constrictor ring was confirmed when the angiogram demonstrated 50\% stenosis (45-52\%) (Figure 1).

Post-operative arterial duplex ultrasound scan was repeated at 7-8 days. Peak systolic velocity ratio (PSV at the stenosis / PSV proximal to the stenosis) of 4 indicates a $70 \%$ stenosis. Confirmation of 70\% canine femoral artery stenosis was obtained when the right femoral angiogram demonstrated an arterial stenosis of $68-72 \%$ at the site of the constrictor ring.

\section{Flow Ultrasound Assessment of Canine Femoral Artery}

All ultrasound assessments were performed with a Mindray Resona 7 ultrasound system (Shenzhen Mindray Bio-Medical Electronics Co., Ltd., Shenzhen, China). A 3-11 MHz linear array transducer (L11-3U) was used for the examination. The ultrasound system was equipped with $\mathrm{V}$ Flow mode, and is capable of obtaining blood flow turbulence index (Tur) at regions of interest (ROI). V Flow uses a series of arrows to represent blood flow direction and velocity. The arrow orientation indicates the direction of blood flow, and the arrow length and color represent the flow velocity. The longer the arrow, the higher the velocity. The range of velocities from high

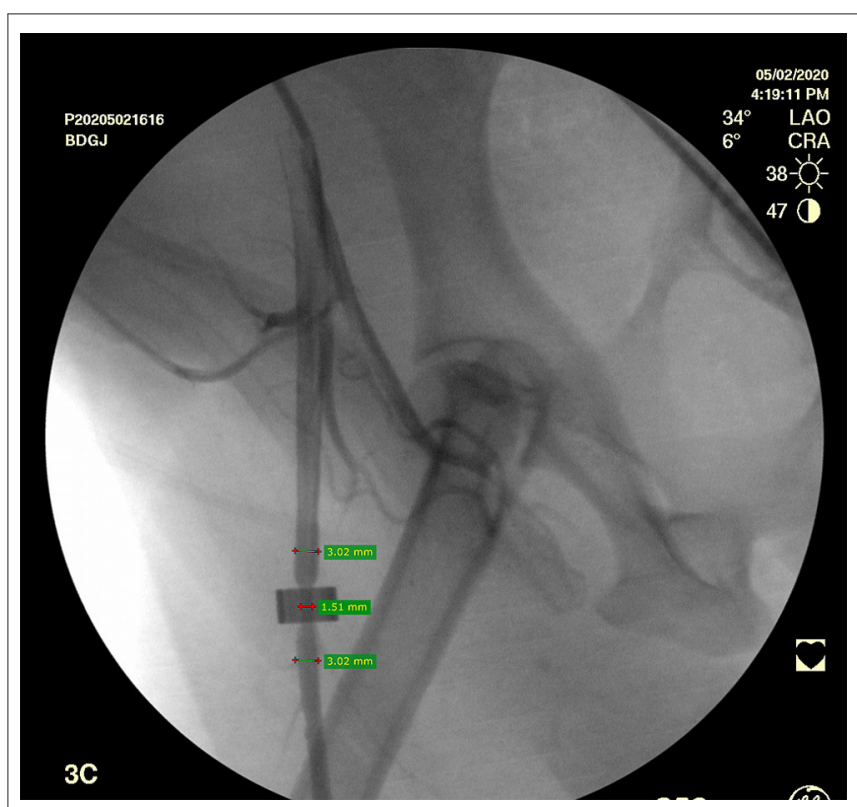

FIGURE 1 | Selective right femoral artery angiogram shows a canine femoral artery stenosis (50\%). 


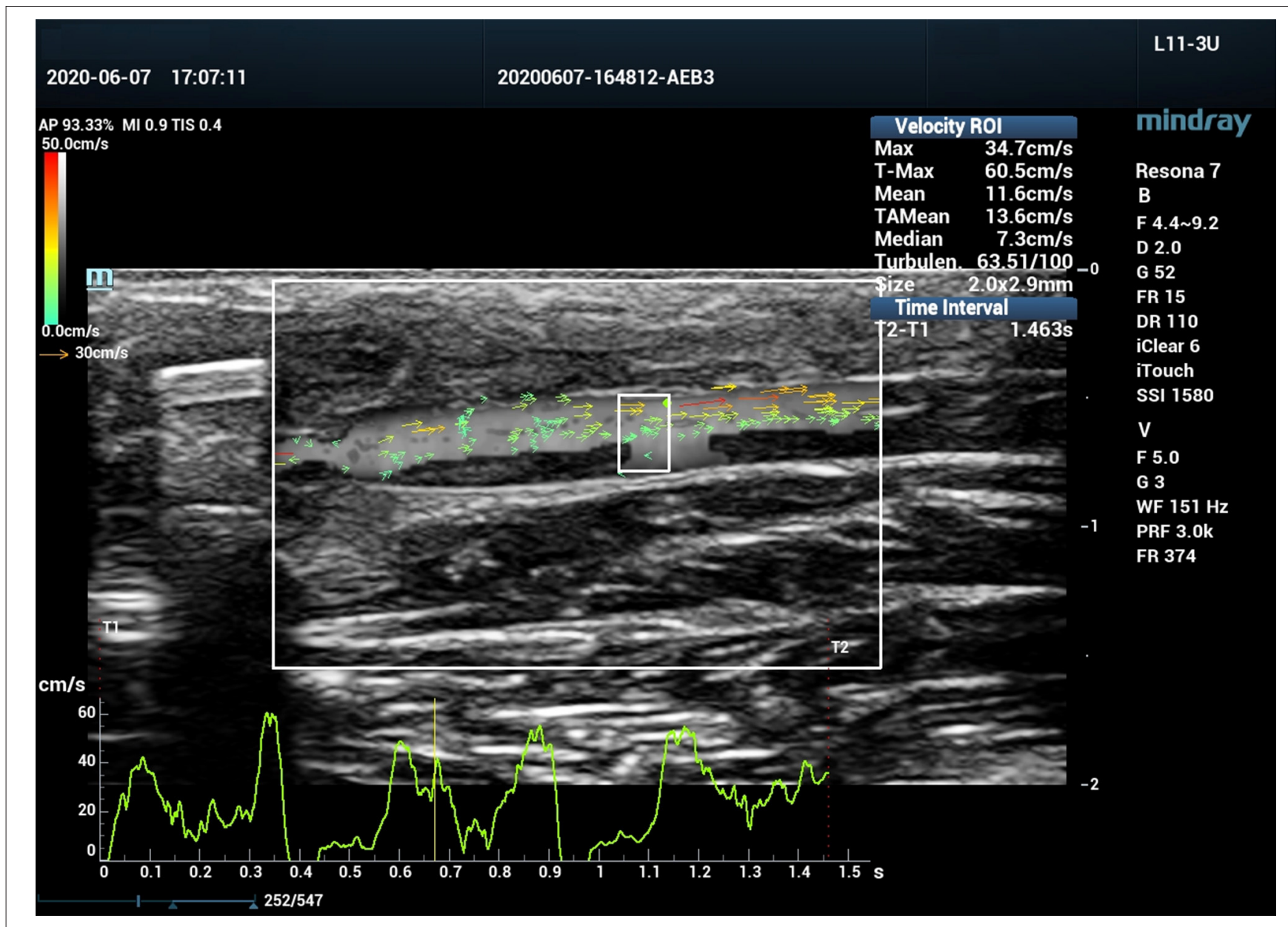

FIGURE 2 | Vector flow imaging (V Flow) of a canine femoral artery.

to low are expressed by colors from red to orange, to yellow, and to green (Figure 2). Tur indices were calculated by the ultrasound system using the following equation:

$$
\operatorname{Tur}=\left(1-\frac{\sqrt{C^{2}+S^{2}}}{N}\right) \times 100 \%
$$

Where

$$
\begin{gathered}
C=\sum_{i=1}^{N} \cos \theta_{i} \\
S=\sum_{i=1}^{N} \sin \theta_{i}
\end{gathered}
$$

$N$ : number of velocity measuring points in the ROI, $\theta_{i}$ : flow velocity angle at $i$ th measuring point, and Tur indices ranged
TABLE 1 | Baseline Tur indices (\%) in 12 canine femoral arteries.

\begin{tabular}{lcccc}
\hline Case No & Tur indices (\%) & Mean & $\begin{array}{c}\text { Standard } \\
\text { deviation }\end{array}$ & $\begin{array}{c}\text { No of cases with Tur } \\
<\mathbf{1 \%}(\%)\end{array}$ \\
\hline 1 & 0.18 & 0.38 & 0.68 & $11(91.7)$ \\
2 & 0.12 & & & \\
3 & 0.24 & & & \\
4 & 0.25 & & \\
5 & 0.99 & & \\
6 & 0.04 & & \\
7 & 0.04 & & \\
8 & 0.11 & & \\
9 & 0.09 & & \\
10 & 2.38 & & \\
11 & 0.07 & & \\
12 & 0.02 & & \\
\hline
\end{tabular}

from 0 to $1(0-100 \%), 0$ indicates pure laminar flow which means that all flow directions are exactly the same. The bigger the Tur is, the more turbulent the flow is (11). 
TABLE 2 | Tur indices (\%) distal to a 50\% stenosis in 12 canine femoral arteries.

\begin{tabular}{|c|c|c|c|c|c|c|c|c|c|c|c|}
\hline Dista & al to stenosis (mm) & 1 & 3 & 5 & 7 & 9 & 11 & 13 & 15 & 17 & 19 \\
\hline \multirow{12}{*}{ Tur (\%) } & No 1 & 3.42 & 4.12 & 2.85 & 2.25 & 1.11 & 0.62 & 0.14 & 0.19 & 0.25 & 0.30 \\
\hline & No 2 & 1.94 & 1.03 & 3.97 & 4.63 & 1.19 & 0.74 & 0.13 & 0.11 & 0.09 & 0.15 \\
\hline & No 3 & 2.49 & 2.16 & 1.45 & 1.94 & 1.35 & 1.17 & 1.56 & 2.52 & 1.24 & 0.33 \\
\hline & No 4 & 14.09 & 9.52 & 12.11 & 18.96 & 13.45 & 0.28 & 0.06 & 2.07 & 0.50 & 0.05 \\
\hline & No 5 & 1.43 & 0.23 & 3.54 & 7.62 & 9.91 & 4.11 & 6.36 & 6.20 & 2.10 & 0.20 \\
\hline & No 6 & 0.87 & 2.31 & 3.69 & 3.46 & 4.89 & 7.11 & 3.12 & 3.89 & 4.65 & 2.22 \\
\hline & No 7 & 8.95 & 15.11 & 19.85 & 24.81 & 19.61 & 10.83 & 3.52 & 0.36 & 3.52 & 3.59 \\
\hline & No 8 & 2.62 & 2.00 & 6.33 & 1.34 & 1.18 & 0.64 & 0.67 & 2.60 & 0.86 & 1.70 \\
\hline & No 9 & 1.77 & 0.28 & 7.36 & 10.60 & 0.40 & 1.88 & 0.62 & 0.35 & 0.09 & 0.52 \\
\hline & No 10 & 8.20 & 17.56 & 7.33 & 8.33 & 3.32 & 0.14 & 0.12 & 0.08 & 0.06 & 0.06 \\
\hline & No 11 & 0.21 & 4.47 & 1.70 & 25.49 & 2.64 & 2.07 & 0.26 & 0.17 & 0.12 & 0.08 \\
\hline & No 12 & 4.03 & 3.61 & 1.63 & 14.75 & 18.63 & 5.64 & 1.88 & 1.19 & 1.09 & 0.14 \\
\hline \multicolumn{2}{|r|}{ Mean } & 4.17 & 5.20 & 5.98 & 10.35 & 6.47 & 2.94 & 1.54 & 1.64 & 1.21 & 0.78 \\
\hline \multicolumn{2}{|c|}{ Standard deviation } & 4.14 & 5.78 & 5.37 & 8.74 & 7.11 & 3.36 & 1.93 & 1.91 & 1.49 & 1.13 \\
\hline \multicolumn{2}{|c|}{ No of cases with Tur <1\% (\%) } & 2 (16.7) & $2(16.7)$ & $0(0)$ & $0(0)$ & $1(8.3)$ & $5(42.7)$ & 7 (58.3) & $6(50.0)$ & 7 (58.3) & 10 (83.3) \\
\hline \multicolumn{2}{|c|}{ No of cases with Tur > 1\% (\%) } & $10(83.3)$ & 10 (83.3) & $12(100)$ & $12(100)$ & $11(91.7)$ & $7(58.3)$ & $5(41.7)$ & $6(50.0)$ & $5(41.7)$ & $2(16.7)$ \\
\hline \multicolumn{2}{|c|}{ No of cases with maximum Tur (\%) } & $0(0)$ & $2(16.7)$ & $1(8.3)$ & $5(41.7)$ & $2(16.7)$ & 1 (8.3) & $0(0)$ & $1(8.3)$ & $0(0)$ & $0(0)$ \\
\hline
\end{tabular}
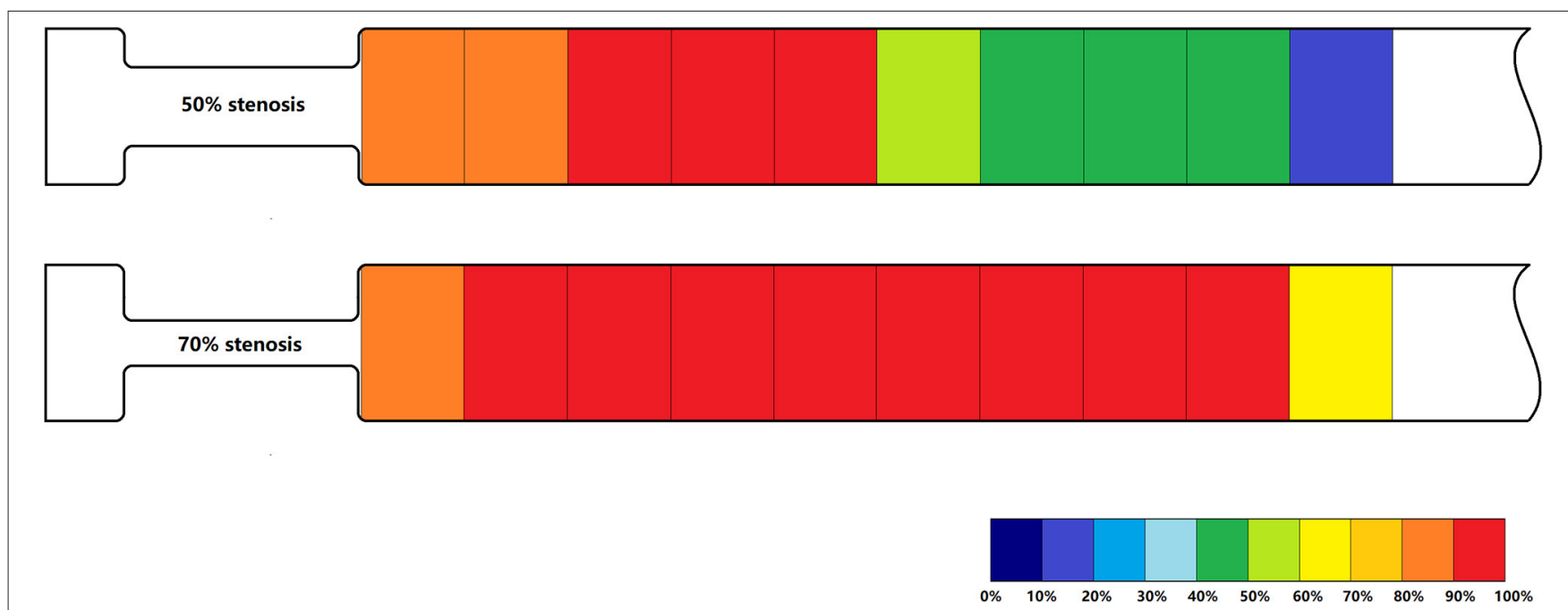

FIGURE 3 | Percentage of Tur indices (>1\%) in relation to distances distal to a $50 \%$ stenosis (above) and a $70 \%$ stenosis (below) in 12 canine femoral arteries.

\section{Flow Acquisition}

V Flow ultrasound images of the right femoral artery in all 12 dogs were recorded preoperatively as a baseline and then after the placement of an ameroid constrictor when the femoral artery stenosis reached $50 \%$ and then $70 \%$. Grayscale ultrasound was used first to display the longitudinal view of the femoral artery. The "V Flow" tab was activated to enter the V Flow mode and $1.5 \mathrm{~s}$ of $\mathrm{V}$ Flow cine loop was captured with image width of $2.4 \mathrm{~cm}$, depth of $1.5 \mathrm{~cm}$ and arrow density of $10 \%$. The heart rate (HR) of the experimental dog was simultaneously recorded.

\section{Tur Index Measurement}

A V Flow cine loop recorded in the ultrasound system was uploaded, and then the "Velocity ROI" box was positioned to the predefined area of the femoral artery for Tur index measurement.
The width of the ROI was $2 \mathrm{~mm}$ and the depth was the internal diameter of the artery. Tur indices were measured preoperatively, and then $1,3,5,7,9,11,13,15,17$, and $19 \mathrm{~mm}$ distal to the femoral artery stenosis at 50\% and then $70 \%$. Export Tur index data of 1.5-s V Flow cine loop ( $\sim 2-3$ cardiac cycles) to a .CVS file containing 500-600 Tur indices, each was from one of 500-600 V Flow frames.

\section{Tur Index Calculation}

Tur indices at different cardiac phases are different even in the same ROI (10) and it is not always possible to determine each frame of the $\mathrm{V}$ Flow image captured from the specific moment of a cardiac cycle. Therefore, the averaged Tur index during one cardiac cycle from the beginning of the recorded V Flow cine loop was calculated for each observation. Number of frames in one 


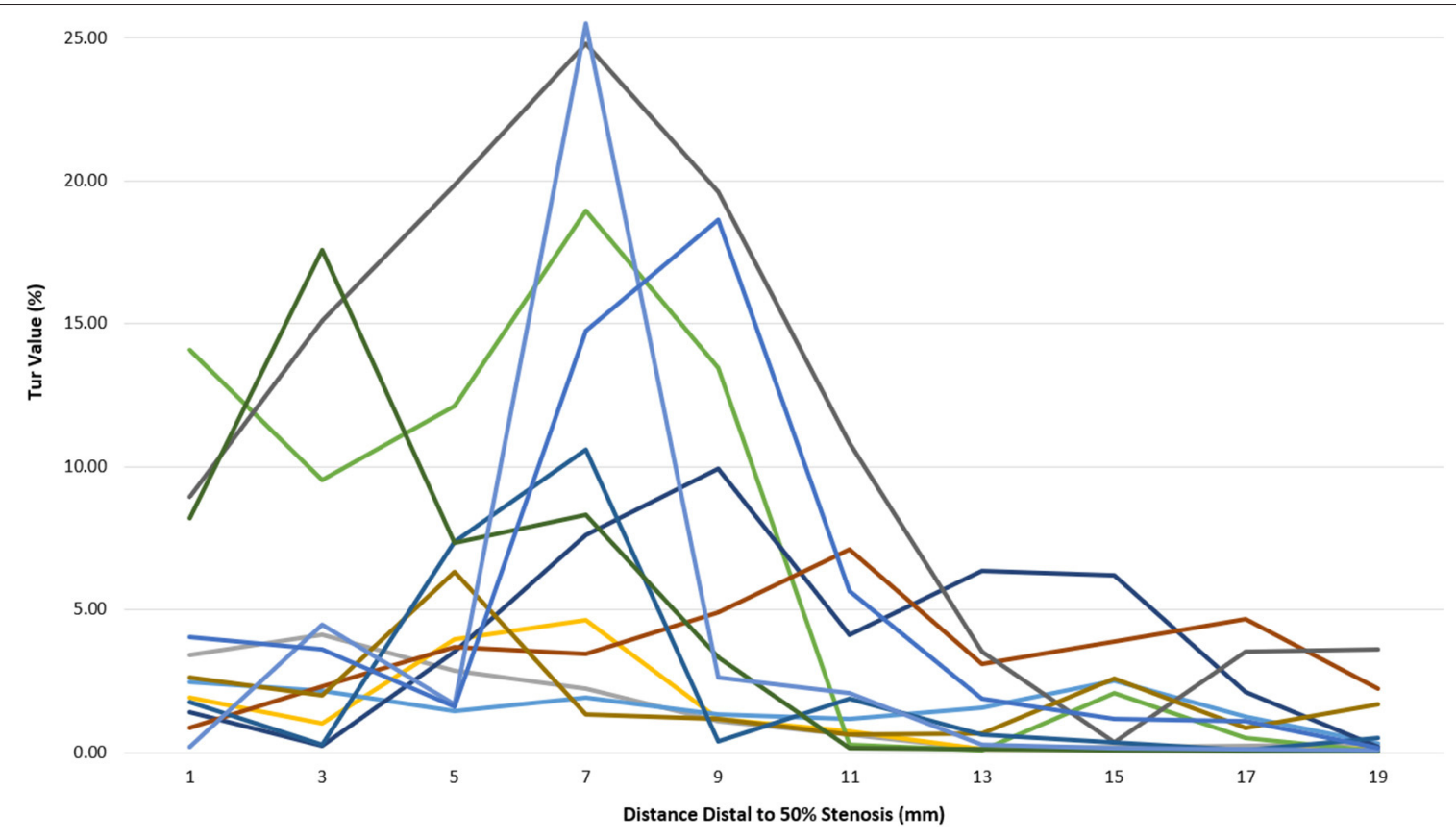

FIGURE 4 | Tur index changes in relation to distance distal to a 50\% stenosis in 12 canine femoral arteries.

TABLE 3 | Tur indices (\%) distal to a 70\% stenosis in 12 canine femoral arteries.

\begin{tabular}{cccccccccccc}
\hline Distance distal to stenosis $(\mathbf{m m})$ & $\mathbf{1}$ & $\mathbf{3}$ & $\mathbf{5}$ & $\mathbf{7}$ & $\mathbf{9}$ & $\mathbf{1 1}$ & $\mathbf{1 3}$ & $\mathbf{1 5}$ & $\mathbf{1 7}$ & $\mathbf{1 9}$ \\
\hline No 1 & 4.19 & 2.34 & 2.58 & 5.83 & 16.95 & 12.30 & 9.66 & 3.21 & 2.15 & 0.91 \\
No 2* & 1.49 & 0.05 & 0.03 & 0.03 & 0.01 & 0.01 & 0.02 & 0.01 & 0.02 & 0.03 \\
No 3 & 0.55 & 4.25 & 18.26 & 20.16 & 13.84 & 4.26 & 4.73 & 2.73 & 9.07 & 2.46 \\
No 4 & 17.08 & 19.59 & 34.29 & 55.76 & 59.48 & 61.97 & 48.49 & 19.83 & 39.47 & 21.31 \\
No 5 & 9.08 & 20.33 & 32.39 & 35.09 & 58.24 & 66.02 & 62.10 & 54.93 & 38.95 & 10.44 \\
No 6 & 9.94 & 31.85 & 41.67 & 42.24 & 20.57 & 2.84 & 2.31 & 2 & 4.01 & 6.96 \\
No 7 & 4.26 & 17.6 & 17.76 & 26.51 & 53.19 & 23.31 & 7.66 & 0.08 & 0.12 & 0.09 \\
No 8 & 7.25 & 24.21 & 56.23 & 67.26 & 56.19 & 39.24 & 39.53 & 32.59 & 23.11 & 1.4 \\
No 9 & 2.73 & 5.68 & 8.60 & 38.97 & 43.89 & 44.08 & 37.16 & 24.57 & 10.38 & 3.78 \\
No 10 & 2.05 & 6.64 & 32.13 & 19.62 & 24.54 & 9.41 & 5.99 & 7.35 & 3.09 & 0.61 \\
No 11 & 1.76 & 2.86 & 5.09 & 5.67 & 41.42 & 13.89 & 2.38 & 27.19 & 1.13 & 0.87 \\
No 12 & 0.31 & 0.33 & 3.74 & 16.68 & 13.43 & 21.15 & 9.26 & 4.02 & 5.32 & 3.32 \\
\hline Mean ${ }^{* *}$ & 5.38 & 12.33 & 22.98 & 30.34 & 36.52 & 27.13 & 20.84 & 16.23 & 12.44 & 4.74 \\
Standard deviation & 5.08 & 10.69 & 17.66 & 19.69 & 18.90 & 22.43 & 21.63 & 17.35 & 14.69 & 6.32 \\
No of cases with Tur $<1 \%(\%)^{* *}$ & $2(18.2)$ & $1(9.1)$ & $0(0)$ & $0(0)$ & $0(0)$ & $0(0)$ & $0(0)$ & $1(9.1)$ & $1(9.1)$ & $4(36.4)$ \\
No of cases with Tur >1\% (\%) & $9(81.8)$ & $10(90.9)$ & $11(100)$ & $11(100)$ & $11(100)$ & $11(100)$ & $11(100)$ & $10(90.9)$ & $10(90.9)$ & $7(63.6)$ \\
No of cases with maximum Tur* & $0(0)$ & $0(0)$ & $1(9.1)$ & $3(27.3)$ & $3(27.3)$ & $4(36.4)$ & $0(0)$ & $0(0)$ & $0(0)$ & $0(0)$ \\
\hline
\end{tabular}

${ }^{*}$ Post-stenotic segment spasm occurred in Case 2.

${ }^{*}$ Case 2 is excluded from calculation.

cardiac cycle $(\mathrm{N})$ is calculated based on the $\mathrm{V}$ Flow frame rate (FR) and the HR at the time:

$$
N=\mathrm{FR} /(\mathrm{HR} / 60)=60 \times \mathrm{FR} / \mathrm{HR}
$$

The mean Tur index from one cardiac cycle was calculated by the sum of Tur indices from the first frame to $N$ th frame divided by $N$.

\section{RESULTS}

Diameters of the right canine femoral arteries in 12 beagle dogs were $2.9-3.1 \mathrm{~mm}$ based on preoperative duplex ultrasound assessment.

Baseline Tur indices obtained from the femoral artery prior to the placement of the ameroid constrictor were shown in Table 1. The Tur index $<1 \%$ was in 11 of 12 arteries $(91.7 \%)$. 


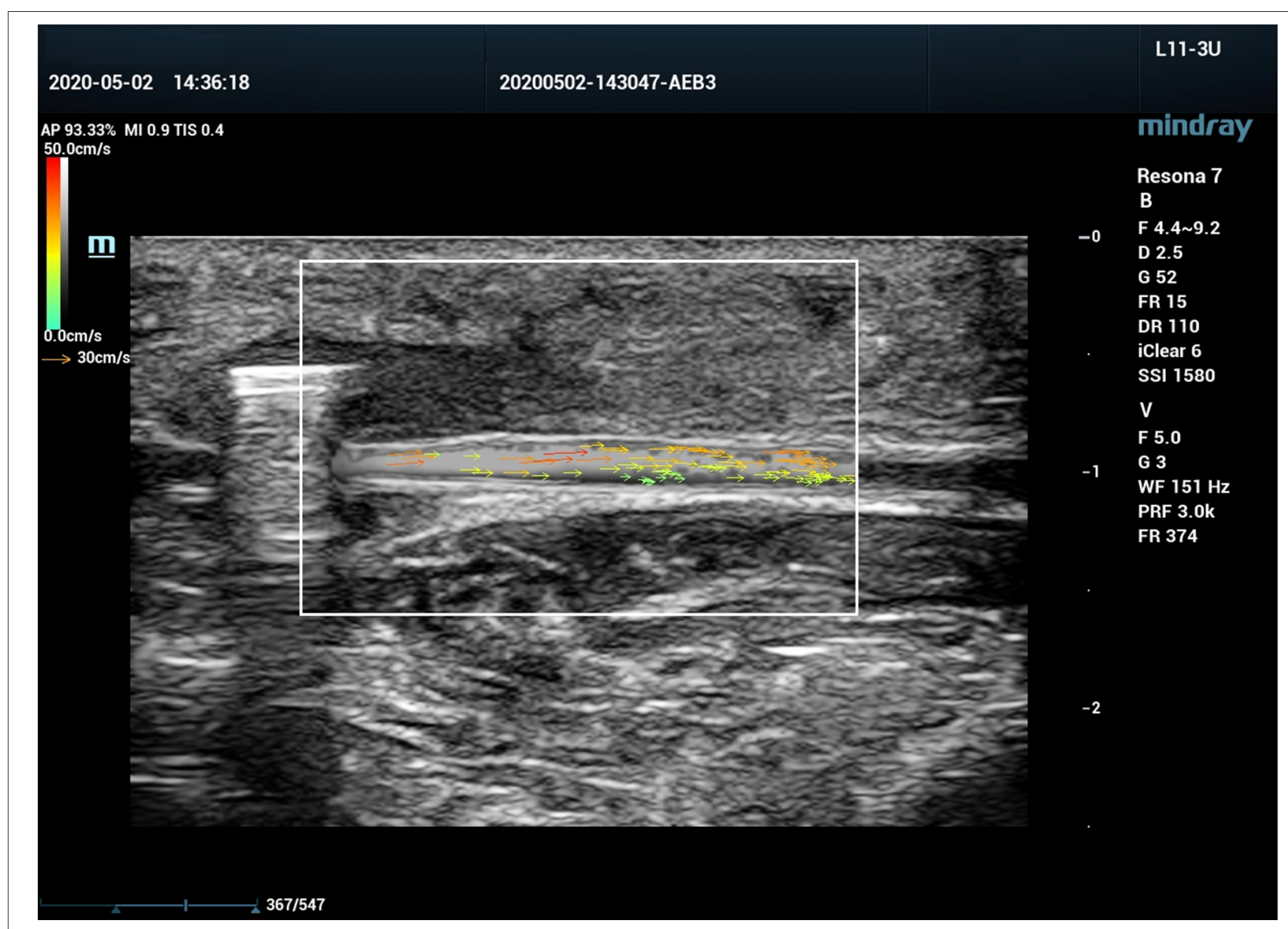

FIGURE 5 | V Flow ultrasound image of a canine femoral artery with post-stenotic segment spasm.

Post-stenotic Tur indices recorded between 1 and $19 \mathrm{~mm}$ distal to a $50 \%$ stenosis following ameroid constrictor placement in 12 canine femoral arteries are shown in Table 2. The Tur index $(>1)$ occurred in $83.3-100 \%$ of the cases between 1 and $9 \mathrm{~mm}$ distal to the stenosis. The number of cases with Tur index (>1\%) decreased to $41.7-58.3 \%$ between 11 and $17 \mathrm{~mm}$ distal to the stenosis, and only $16.7 \%$ at $19 \mathrm{~mm}$ (Table 2 and Figure 3 ). Figure 4 shows Tur index changes in relation to distances distal to a $50 \%$ stenosis. Based on a canine femoral artery diameter of $3 \mathrm{~mm}$, post-stenotic Tur index increased immediately distal to the stenosis and reached its maximum value at $5-11 \mathrm{~mm}$ or $1.7-$ 3.7 diameters downstream in 9 of 12 cases (75\%) with most at $7 \mathrm{~mm}$ or 2.3 times the vessel diameter downstream (5/12). The Tur index decreased sharply afterwards (Table 2 and Figure 4).

Post-stenotic Tur indices distal to a $70 \%$ stenosis are shown in Table 3. Post-stenotic segment spasm occurred in Case 2. The PSVs in the segment were ranged from $128 \mathrm{~cm} / \mathrm{s}$ to $198 \mathrm{~cm} / \mathrm{s}$, and the Tur index $(<1 \%)$ occurred in 9 of 10 measurement sites (Table 3 and Figure 5). This case is not included in the calculation for mean, standard deviation, number of cases with Tur $<1 \%$, number of cases with Tur $>1 \%$, and number of cases with maximum Tur. In the remainder of 11 cases, the Tur index $(>1 \%$ ) was $81.8-100 \%$ between 1 and $17 \mathrm{~mm}$ distal to the stenosis, and $63.6 \%$ at $19 \mathrm{~mm}$ (Table 3 and Figure 3). Figure 6 shows Tur index changes in relation to distances distal to a $70 \%$ stenosis. Based on a canine femoral artery diameter of $3 \mathrm{~mm}$, post-stenotic Tur index increased immediately distal to the stenosis and reached its maximum value at $5-11 \mathrm{~mm}$ or $1.7-$ 3.7 diameters downstream in all 11 cases (100\%) followed by a decrease (Table 3 and Figure 6).

Figure 7 displays mean Tur index changes distal to a $50 \%$ stenosis (solid line) and a 70\% stenosis (dot line). It appears that post-stenotic Tur index increases were higher and lasted longer in arteries with a $70 \%$ stenosis than those with a $50 \%$ stenosis. It is needed to point out that Tur index values in both groups had a large standard deviation (see error bars in the figure).

\section{DISCUSSION}

Blood flow in a straight artery with near uniform diameters is generally considered to be close to laminar flow (22). Nonlaminar flow in an artery can be observed when it becomes 


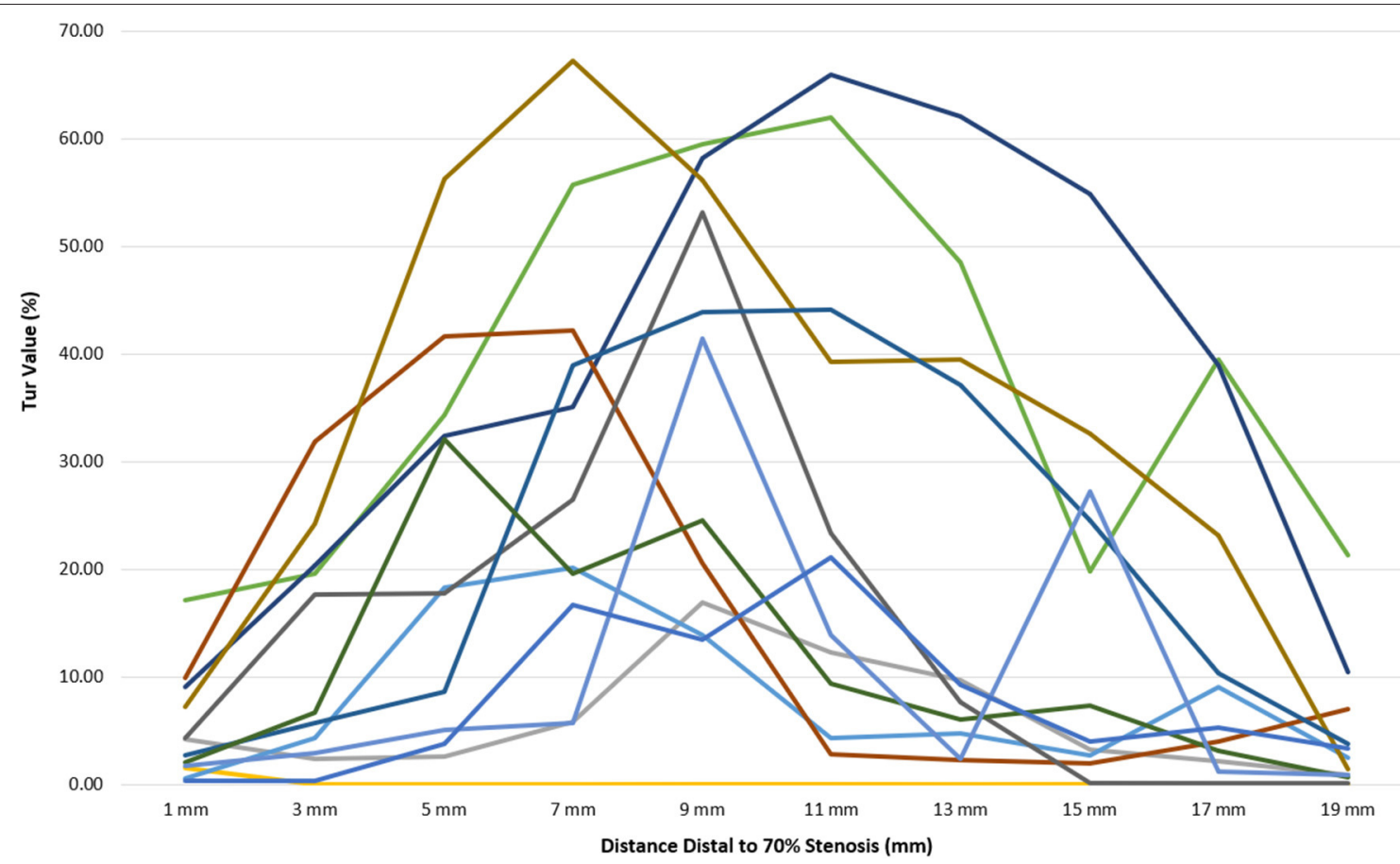

FIGURE 6 | Tur index changes in relation to distance distal to a 70\% stenosis in 12 canine femoral arteries.

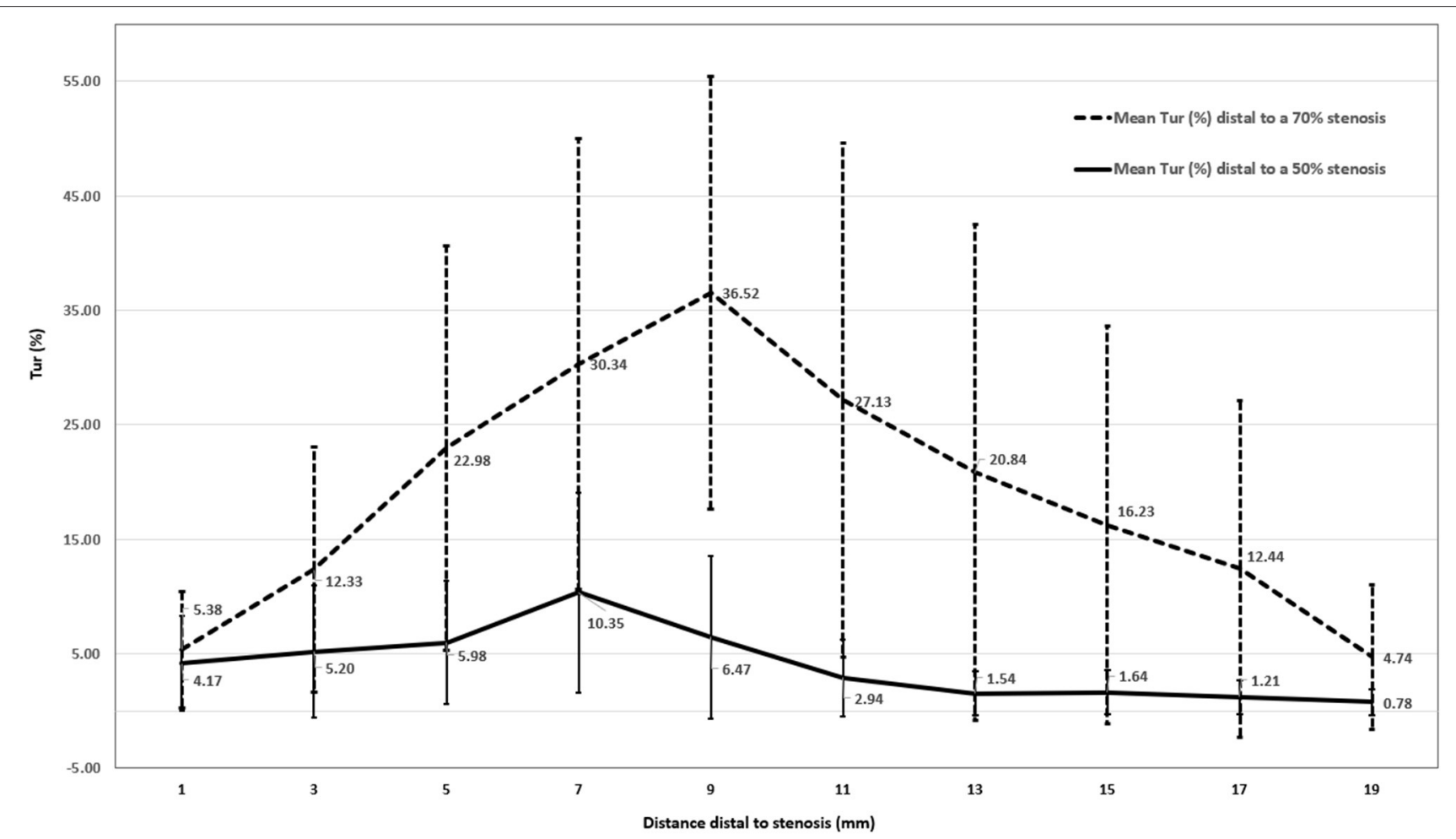

FIGURE 7 | Mean Tur index changes distal to a $50 \%$ stenosis $(N=12)$ and a $70 \%$ stenosis $(N=11)$ in canine femoral arteries (error bars represents standard deviation of the means). 
bifurcated, dilated, tortuous, downstream to a stenosis, or has an arteriovenous communication (23). It is believed that poststenotic turbulence is related to post-stenotic dilation and wall shear stress (24). Since blood behaves as a non-Newtonian fluid and changes its viscosity when flow velocity changes, this further complicates flow turbulence, which has been investigated by a series of numerical studies (25-28). Evaluation of post-stenotic flow turbulence is important in the diagnosis of arterial stenosis, and included in many duplex ultrasound diagnostic criteria $(8,29,30)$. The study of flow turbulence is also helpful in understanding of the mechanism of formation and progression of vascular disorders as well as in the design of medical devices that mimic or alter blood flow (31).

Conventional ultrasound systems are able to measure blood flow velocities with spectral Doppler and observe flow direction with color Doppler imaging. Spectral Doppler and color Doppler are based on Doppler principle and angledependency. Therefore, Doppler ultrasound only estimates the axial component of flow velocity and is limited by the vessel geometry. When flow turbulence is present in an artery, blood flows in multiple directions. These ultrasound systems can only provide qualitative information regarding flow turbulence by multiple colors on color Doppler imaging and spectral broadening on pulsed wave (PW) Doppler spectrum representing various flow velocities and multiple flow directions. However, evaluations of flow turbulence by both color Doppler imaging and PW Doppler spectrum are qualitative and have their limitations. If the color velocity scale is set below the mean velocity of blood flow, aliasing may appear throughout the vessel lumen that makes it difficult to identify flow turbulence on color Doppler. On the PW Doppler spectrum, spurious spectral broadening can result from a large Doppler angle, a large sample volume $(>3.5 \mathrm{~mm})$, a sample volume located close to the vessel wall, or a high PW Doppler gain setting (32). V Flow uses multi-directional ultrasound transmission and reception (9) based on plane wave and focus wave interleaved scanning (11). The actual direction of the velocity is reconstructed by the velocity components from different directions. Therefore, it is capable of visualizing and measuring flow velocities in all directions (14). The Tur index measurement makes it possible to assess flow turbulence quantitatively $(11,12)$. However, it is not fully understood how to apply the Tur index in the assessment of flow turbulence and its clinical significance.

Theoretically, pure laminar flow travels in parallel layers with exactly the same flow directions and the Tur index equals to $0 \%$ while most extra turbulent flow with opposite direction flow has a Tur index of $100 \%(11,12)$. In this study, Tur index was $<1 \%(0.02-0.99 \%)$ in 11 of 12 normal canine femoral arteries $(91.7 \%)$. Tur index (>1\%) was observed immediately distal to an arterial stenosis, in 10 of 12 cases (83\%) distal to a $50 \%$ stenosis and 9 of 11 cases (82\%) distal to a $70 \%$ stenosis. The Tur index peaked within 1.7-3.7 diameters downstream in 9 of 12 cases $(75 \%)$ to a $50 \%$ stenosis and in all 11 cases $(100 \%)$ to a $70 \%$ stenosis. At $19 \mathrm{~mm}$ or 6.3 diameters downstream to a stenosis, the Tur index recovered to $<1 \%$ in more cases with a less significant stenosis (50\%) than those with a more significant stenosis (70\%), being $83.3 \%(10 / 12)$ vs. $36.4 \%(4 / 11)$.

There is no published threshold of the Tur index for distinguishing turbulent flow from normal flow in a straight artery. This study attempts to explore such a threshold. Tur index of $1 \%$ seems a useful threshold for assessment of flow turbulence in this small sample study. Further studies are needed to determine clinically significant thresholds for various clinical applications. Post-stenotic Tur index values had a large standard deviation in arteries with a $50 \%$ stenosis and with a $70 \%$ stenosis. Therefore, it is less meaningful to compare the mean Tur of the two groups. Nevertheless, it seems arteries with a more severe stenosis had higher and longer lasting post-stenotic Tur index values than those with a less severe stenosis.

It should be pointed out that the size of the canine femoral arteries studied was approximately $3 \mathrm{~mm}$ in diameter and much smaller than those most commonly studied human arteries such as carotid and femoropopliteal arteries. Haemodynamic changes including its response to a stenosis in those arteries might be different.

\section{Limitations}

There are limitations in this study. Small sample size is an obvious one. Tur indices are not the same for the same ROI at different cardiac phases (11). When a stenosis is present, it is not always possible to determine the precise cardiac phase of a Tur index recorded based on the V Flow velocity waveform. Therefore, Tur index data presented in this study are the averaged Tur indices representing all Tur indices during one cardiac cycle for each observation rather than individual instantaneous values, and may not be the optimal data for the evaluation of post-stenotic flow turbulence. Tur index is a completely direction-based quantification number for turbulent flow. The magnitude information of the velocity has not been considered in quantifying the flow turbulence even though the velocities at different locations within the ROI are different. Current V Flow uses two-dimensional (2D) technology and the Tur index is based on $2 \mathrm{D}$ data rather than three-dimensional (3D) data while actual post-stenotic turbulence occurs in a $3 \mathrm{D}$ space. A $3 \mathrm{D} \mathrm{V}$ Flow in the future will deliver a better representation of complex haemodynamic patterns (16).

\section{CONCLUSION}

This limited study demonstrates the mean Tur index in a cardiac cycle is generally $<1 \%$ in a straight artery segment without a stenosis. Flow turbulence indicated by increase in the Tur index occurred immediately distal to a stenosis (50 or 70\%). The Tur value reaches its peak in the artery around 2.3-3.7 diameters downstream to a stenosis and recovered to $<1 \%$ earlier in arteries with a less significant stenosis than those with a more significant stenosis. V Flow can provide additional flow turbulence information when assessing arterial stenosis with ultrasound. Further studies with larger sample size are needed to evaluate the value of V Flow in clinical applications. 


\section{DATA AVAILABILITY STATEMENT}

The raw data supporting the conclusions of this article will be made available by the authors, without undue reservation.

\section{ETHICS STATEMENT}

The animal study was reviewed and approved by the Experimental Animal Welfare and Ethics Committee, the Fourth Medical Center of PLA General Hospital.

\section{AUTHOR CONTRIBUTIONS}

CW and YT proposed the scientific problems. CW and RZ designed the experiments. RZ, HZ, and WW carried out the animal experiments. RZ and $\mathrm{YT}$ processed and

\section{REFERENCES}

1. Peach G, Griffin M, Jones KG, Thompson MM, Hinchliffe RJ. Diagnosis and management of peripheral arterial disease. BMJ. (2012) 345:36-41. doi: 10.1136/bmj.e5208

2. Abdulhannan P, Russell DA, Homer-Vanniasinkam S. Peripheral arterial disease: a literature review. Br Med Bull. (2012) 104:21-39. doi: $10.1093 / \mathrm{bmb} / \mathrm{lds} 027$

3. Weinberg I, Jaff MR. Nonatherosclerotic arterial disorders of the lower extremities. Circulation. (2012) 126:213-22. doi: 10.1161/CIRCULATIONAHA.111.060335

4. Seifert H, Jäger K. Diagnostic value of duplex scanning in peripheral vascular disease. Vasc Med Rev. (1990) 1:21-33. doi: 10.1177/1358836X9000100103

5. Gerhard-Herman M, Gardin JM, Jaff M, Mohler E, Roman M, Naqvi $\mathrm{TZ}$, et al. Guidelines for noninvasive vascular laboratory testing: a report from the American Society of Echocardiography and the Society of Vascular Medicine and Biology. J Am Soc Echocardiogr. (2006) 19:955-72. doi: 10.1016/j.echo.2006.04.019

6. Gupta P, Lyons S, Hedgire S. Ultrasound imaging of the arterial system. Cardiovasc Diagn Ther. (2019) 9(Suppl 1):S2-13. doi: 10.21037/cdt.2019.02.05

7. AIUM practice parameter for the performance of peripheral arterial ultrasound examinations using color and spectral Doppler imaging. $J$ Ultrasound Med. (2021) 40:E17-24. doi: 10.1002/jum.15643

8. Kim ES, Sharma AM, Scissons R, Dawson D, Eberhardt RT, GerhardHerman $\mathrm{M}$, et al. Interpretation of peripheral arterial and venous Doppler waveforms: A consensus statement from the Society for Vascular Medicine and Society for Vascular Ultrasound. Vasc Med. (2020) 25:484-506. doi: 10.1177/1358863X20937665

9. Yiu BYS, Lai SSM, Yu ACH. Vector projectile imaging: time-resolved dynamic visualization of complex flow patterns. Ultrasound Med Biol. (2014) 40:2295309. doi: 10.1016/j.ultrasmedbio.2014.03.014

10. Goddi AG, Fanizza M, Bortolotto C, Raciti MV, Fiorina I, He X, et al. Vector flow imaging techniques: An innovative ultrasonographic technique for the study of blood flow. J Clin Ultrasound. (2017) 45:582-8. doi: $10.1002 /$ jcu. 22519

11. Du Y, Shen Y, Yiu BYS, Yu ACH, Zhu L. High frame rate vector flow imaging: Development as a new diagnostic mode on a clinical scanner. In: Kobe: 2018 IEEE International Ultrasonics Symposium (IUS). (2018). p. 1-4. doi: 10.1109/ULTSYM.2018.8580187

12. Du Y, Zhu L, He X, Feng H. [Quantitative ultrasound measurements of blood flow velocity and turbulence]. Zhongguo Yi Liao Qi Xie Za Zhi. (2018) 42:157-60. doi: 10.3969/j.issn.1671-7104.2018.03.001

13. Pedersen MM, Pihl MJ, Haugaard P, Hansen KL, Lange T, Lönn L, et al. Novel flow quantification of the carotid bulb and the common carotid artery with vector flow ultrasound. Ultrasound Med Biol. (2014) 40:2700-6. doi: 10.1016/j.ultrasmedbio.2014.06.001 calculated the data. YT and RZ conducted the statistical analysis and wrote the draft manuscript. YD provided technical support for the experiment and manuscript writing. All authors contributed to the article and approved the submitted version.

\section{FUNDING}

This work was supported by the National Natural Science Foundation of China (Grant No. 81771833) and the Beijing Natural Science Foundation (Grant No. 7172209).

\section{ACKNOWLEDGMENTS}

The authors greatly thank Mrs. Jayne Chambers for her proofreading of the manuscript.

14. Goddi A, Bortolotto C, Fiorina I, Raciti MV, Fanizza M, Turpini E, et al. High frame rate vector flow imaging of the carotid bifurcation. Insights Imaging (2017) 8:319-28. doi: 10.1007/s13244-017-0554-5

15. Goddi A, Bortolotto C, Raciti MV, Fiorina I, Aiani L, Magistretti G, et al. High-frame rate vector flow imaging of the carotid bifurcation in healthy adults: comparison with color doppler imaging. J Ultrasound Med. (2018) 37:2263-75. doi: 10.1002/jum.14579

16. Du Y, Goddi A, Bortolotto C, Shen Y, Dell'Era A, Calliada F, et al Wall shear stress measurements based on ultrasound vector flow imaging: Theoretical studies and clinical examples. J Ultrasound Med. (2020) 39:164964. doi: 10.1002/jum. 15253

17. Saito K, Abe S, Kumamoto M, Uchihara Y, Tanaka A, Sugie K, et al. Blood flow visualization and wall shear stress measurement of carotid arteries using vascular vector flow mapping. Ultrasound Med Biol. (2020) 46:2692-9. doi: 10.1016/j.ultrasmedbio.2020.06.018

18. Brandt AH, Nguyen $\mathrm{T}$, Gutte $\mathrm{H}$, Carlsen JF, Moshavegh R, Jensen JA, et al. Carotid stenosis assessment with vector concentration before and after stenting. Diagnostics. (2020) 10:420. doi: 10.3390/ diagnostics 10060420

19. Brandt AH, Olesen JB, Ramin Moshavegh R, Jensen JA, Nielsen MB, Hansen KL, et al. Common carotid artery volume flow: A comparison study between ultrasound vector flow imaging and phase contrast magnetic resonance imaging. Neurol Int. (2021) 13:269-78. doi: 10.3390/ neurolint 13030028

20. Qiu Y, Yang D, Zhang Q, Chen K, Dong Y, Wang WP, et al. V Flow technology in measurement of wall shear stress of common carotid arteries in healthy adults: feasibility and normal values. Clin Hemorheol Microcirc. (2020) 74:453-62. doi: $10.3233 / \mathrm{CH}-19$ 0719

21. Qiu Y, Dong Y, Mao F, Zhang Q, Yang D, Chen K, et al. High-frame rate vector flow imaging technique: initial application in evaluating the hemodynamic changes of carotid stenosis caused by atherosclerosis. Front Cardiovasc Med. (2021) 8:617391. doi: 10.3389/fcvm.2021.617391

22. Klabunde RE. Cardiovascular Physiology Concepts. 3rd ed. Philadelphia, PA: Wolters Kluwer (2021).

23. Owen CA, Roberts M. Arterial vascular hemodynamics. JDMS. (2007) 23:12940. doi: $10.1177 / 8756479307302338$

24. Ojha M, Johnston KW, Cobbold RS. Evidence of a possible link between poststenotic dilation and wall shear stress. J Vasc Surg. (1990) 11:127-35.

25. Bit A, Chattopadhyay $H$. Numerical investigations of pulsatile flow in stenosed artery. Acta Bioeng Biomech. (2014) 16:33-44. doi: 10.5277/ABB-00029-2014-05

26. Bit A, Ghagare D, Rizvanov AA, Chattopadhyay H. Assessment of influences of stenoses in right carotid artery on left carotid artery using wall stress marker. BioMed Res Int. (2017) 2017:1-13. doi: 10.1155/2017/293 5195 
27. Bit A, Alblawi A, Chattopadhyay H, Quais QA, Benim AC, Rahimi-Gorji $\mathrm{M}$, et al. Three dimensional numerical analysis of hemodynamic of stenosed artery considering realistic outlet boundary conditions. Comput Methods Programs Biomed. (2020) 185:105163. doi: 10.1016/j.cmpb.2019.105163

28. Bit A, Chattopadhyay H. Assessment of rheological models for prediction of transport phenomena in stenosed artery. Progress Comput Fluid Dyn Int J. (2014) 14:363-74. doi: 10.1504/pcfd.2014.065468

29. Gerhard-Herman M, Gardin JM, Jaff M, Mohler E, Roman M, Naqvi TZ, et al. Guidelines for noninvasive vascular laboratory testing: a report from the American Society of Echocardiography and the Society for Vascular Medicine and Biology. Vasc Med. (2006) 11:183-200. doi: 10.1177/1358863x06070516

30. Sprynger M, Rigo F, Moonen M, Aboyans V, Edvardsen T, de Alcantara $\mathrm{ML}$, et al. Focus on echovascular imaging assessment of arterial disease: complement to the ESC guidelines (PARTIM 1) in collaboration with the Working Group on Aorta and Peripheral Vascular Diseases. Eur Heart J Cardiovasc Imaging. (2018) 19:1195-221. doi: 10.1093/ehjci/jey103

31. Ku DN. Blood flow in arteries. Ann Rev Fluid Mech. (1997) 29:399-434. doi: 10.1146/annurev.fluid.29.1.399

32. Tahmasebpour HR, Buckley AR, Cooperberg PL, Fix CH, et al. Sonographic examination of the carotid arteries. Radiographics. (2005) 25:1561-75. doi: $10.1148 /$ rg. 256045013
Conflict of Interest: YD is employed by Shenzhen Mindray Bio-Medical Electronics Co., Ltd., Shenzhen, China.

The remaining authors declare that the research was conducted in the absence of any commercial or financial relationships that could be construed as a potential conflict of interest.

Publisher's Note: All claims expressed in this article are solely those of the authors and do not necessarily represent those of their affiliated organizations, or those of the publisher, the editors and the reviewers. Any product that may be evaluated in this article, or claim that may be made by its manufacturer, is not guaranteed or endorsed by the publisher.

Copyright (c) 2022 Zhao, Zheng, Wang, Du, Tong and Wen. This is an open-access article distributed under the terms of the Creative Commons Attribution License (CC $B Y)$. The use, distribution or reproduction in other forums is permitted, provided the original author(s) and the copyright owner(s) are credited and that the original publication in this journal is cited, in accordance with accepted academic practice. No use, distribution or reproduction is permitted which does not comply with these terms. 